\title{
Energy Efficient Cross-Layer Approach for Wireless Sensor Networks
}

\author{
Md. Imran Hossain Jony \\ Department of EEE \\ Islamic University of Technology, Gazipur, Dhaka
}

\author{
Mohammad Rakibul Islam \\ Professor, Department of EEE \\ Islamic University of Technology, Gazipur, Dhaka
}

\begin{abstract}
Energy minimization has become a burning issue for Wireless sensor networks (WSNs) which are mainly event based systems and rely on the collective effort of several microsensor nodes continuously observing a physical phenomenon. Energy efficient approaches or tools are the key to prolong the lifetime of the sensor nodes. This paper presents a cross-layer approach between the medium access control (MAC) and the network layer to achieve energy efficiency. Ad-hoc OnDemand Distance Vector (AODV) is used here as routing protocol in the network layer along with IEEE-802.11 protocol in the MAC layer. Simulation results show that cross-layer approach obtains significant energy savings compared with traditional approaches.
\end{abstract}

\section{Keywords}

Wireless sensor network, Cross-layer, MAC, AODV, Energy Consumption, Goodput, Throughput.

\section{INTRODUCTION}

\subsection{Wireless sensor networks}

Wireless sensor networks (WSNs) are getting popular day-byday for applicability, reliability and flexibility to implement in any environment. These are mainly event-based systems that exploit the collective effort of densely deployed micro-sensor nodes which continuously observe certain physical phenomenon. The main objective of any WSN application is to detect event features from the collective information provided by sensor nodes. In a typical sensor network, information collected by multiple local sensors need to be transmitted to a remote central processor. If the remote processor is far away from the source node then the information will first be transmitted to a relay node and then multi hop-based routing will be used to forward the data to its final destination. The main challenge for achieving this objective is mainly posed by the severe energy and processing constraints of low-end wireless sensor nodes as those are battery operated. Therefore, conserving the energy of those sensor nodes has become the most popular research area for the developers to enhance the lifetime of the nodes.

There are lots of applications of sensor nodes which require constant monitoring and detection of specific events. One of the major fields of application of sensor nodes is military including battlefield surveillance and monitoring, detection of attack by weapons of mass destruction, such as chemical, biological, or nuclear and guidance systems of intelligent missiles. Sensors can also be used in environmental applications such as fire and flood detection, habitat exploration of animals. Sensors can be extremely useful in diagnosis of diseases and monitoring the patients. Small sensor devices can be used in the patient's body to monitor their physiological data such as blood pressure or heart rate. These data collected from the sensors can be used from a SCADA (Supervisory Control and Data Acquisition) system to alert the concerned doctor on detection of an anomaly. Such systems provide patients a greater freedom of movement instead of their being confined to a hospital. Therefore, the patents need not to be confined themselves to the boundary of the hospitals, rather they can move anywhere with freedom and relief.

Sensors are also getting popular in commercial applications at home and in industries. Smart sensor nodes are used inside the air conditioners to sense the temperature of a room and then reacted according to the signal sent by remote controllers handled by the user. Sensors can be placed inside several home appliances like ovens, refrigerators, and vacuum cleaners to interact with each other and be remote-controlled. A "smart environment" can be created inside the home which adapts itself according to the user's needs or tastes. For instance, almost everything in the room such as, the lighting, music, and ambiance can be automatically set or controlled according to the user's preferences.

Similar type of control can also be useful in office buildings too. The airflow and temperature of different parts of the building can be automatically controlled using sensor nodes. The inventory control system of warehouses could be improved by installing sensors on the products to track their movement. Therefore it can be summarized that the applications of wireless sensor networks are endless, limited only by the human imagination. Considering all of these huge applications and usefulness of wireless sensor nodes, it has become a serious issue to conserve the battery power of the sensors to prolong their lifetime.

\subsection{Related Work}

Several methods are proposed to save the energy of the sensor nodes. Spatial-temporal correlation is one of those. It has been shown in [1] that exploiting the spatial and temporal correlation improves energy efficiency of communication in WSN. In [2], the authors analyze that the event-to-sink reliable transport (ESRT) protocol which ensures reliable event detection with minimum energy expenditure. Directed diffusion based network are application-aware which enables diffusion to achieve energy savings by selecting empirically good paths and by caching and processing data in-network (e.g., data aggregation) [3]. In [4], the authors show that reception based forwarding strategies are more efficient than purely distance-based strategies. They also show that relative blacklisting schemes reduce disconnections and achieve 


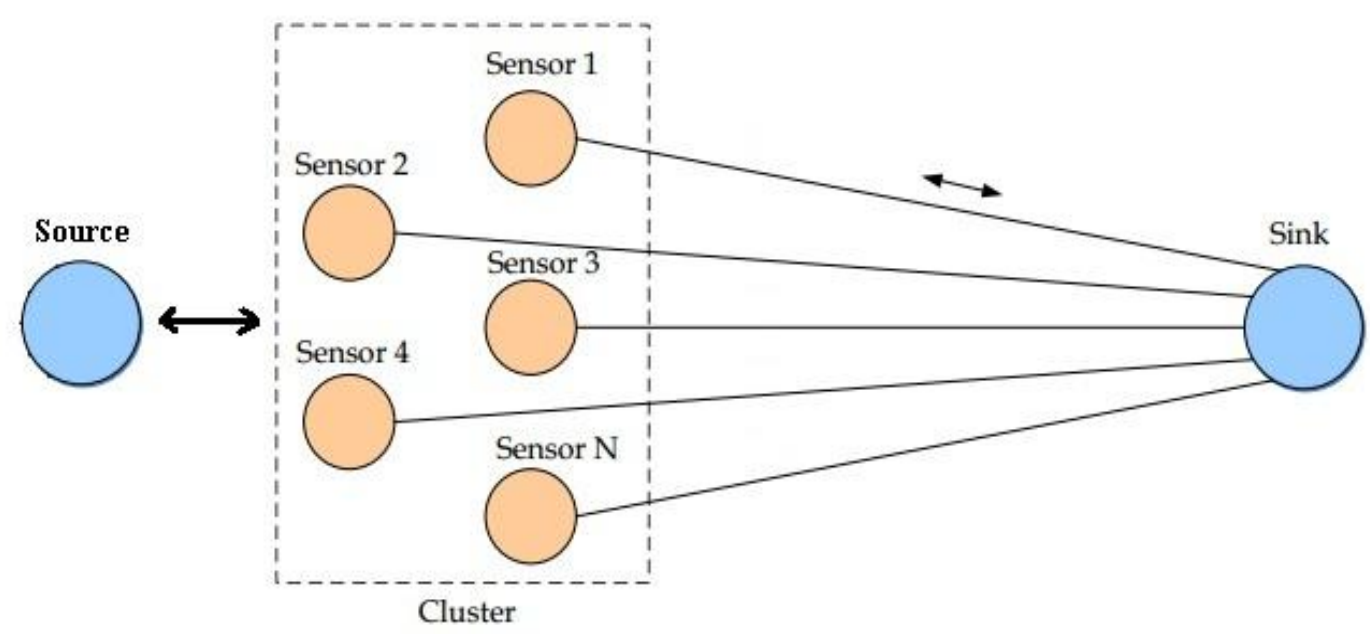

Fig 1: System Model

higher delivery rates than absolute blacklisting schemes and that Automatic Repeat Request (ARQ) schemes become more important in larger networks.

Several works on WSN reveals that cross-layer integration and design techniques also result in significant improvement in terms of energy conservation [5], [6]. The energy consumption for medium access control (MAC) and physical layer is analyzed in [7-8]. In [9], the joint optimal design of the physical, MAC and routing layer is considered which is called as Energy Optimization Approach (EOA). A crosslayer approach is also established between MAC layer and network layer where the grid-quorum system is used on MAC layer and on the network layer the authors propose to find query paths based on the power cost incurred by grid quorums used by nodes along a path [10]. A unified cross-layer protocol (XLP) is presented in [11-12] which achieves congestion control, routing and medium access control in a cross-layer fashion. XLP integrates functionalities of physical to transport layer into a cross-layer protocol using the concept of initiative determination which proves to be more energy efficient than the previous protocols. Initiative determination enables receiver-based contention, initiative-based forwarding, local congestion control and distributed duty cycle operation. All of those methods basically focus on the cross-layer protocols rather than using any existing protocol to implement the cross-layer approach. The focus of us is kept on this issue and the method proposed here uses the technique of using Ad-hoc On-Demand Distance Vector (AODV) protocol inside the MAC layer which reduces the complexity of implementing a new protocol combining several layers.

\subsection{Algorithm}

In this paper, an approach to execute cross-layer between MAC layer and network layer is established which achieves energy efficiency more than a traditional layered approach. The interfacing between these two layers has been strengthened to pass the routing table from network to MAC layer. At first the identity of the routing table of network layer is characterized by a symbol. Then the symbol is called upon from the MAC layer. Therefore, the MAC layer decides whether a signal needs to be sent to the upper layers or not (for receiving node) and to the specific sensor node or not (for transmitting node). Combination of these two processes reduces wastage of energy throughout the network by ensuring more synchronized and efficiently scheduled data transmission and reception.

Among several MAC layer protocols, IEEE 802.11 MAC seemed to be inefficient comparing several other MAC layer protocols. It has been shown that IEEE 802.11 MAC still can be efficient if our cross-layer approach is imposed on it. Our results also outperform S-MAC in several extents [13]. SMAC utilizes coordinated adaptive sleeping where the concept of duty cycle is initialized to keep sensor nodes active for a certain period of time to save energy. The remainder of the paper is organized as follows. Basics and overview of our cross-layer approach are introduced in Section 2. In Section 3, performance evaluations of our approach cross-layer approach are provided along with a comparative analysis. Finally, the paper is concluded in Section 4.

\section{BASICS AND OVERVIEW}

The system considered in our approach is a clustered wireless sensor network shown in Figure 1. Communication link connecting two wireless sensor nodes can in general be Multiple Input Multiple Output (MIMO), Multiple Input Single Output (MISO), Single Input Multiple Output (SIMO) or Single Input Single Output (SISO). We first need to look into the MAC layer along with network layer. The protocols used to determine who goes next on a multi-access channel belong to a sub-layer of the data link layer which is called the MAC sub-layer. Therefore it determines the ability of a node to efficiently share the wireless medium with the other nodes in the network. One of the main objectives of the MAC layer is to keep the energy consumption low by turning off the radio module as often as possible.

In order to design energy aware MAC protocols, the main causes of energy consumption need to be taken into consideration and which are idle listening, overheads, overhearing and collisions presented in [14]. Therefore, these factors need to be minimized in order to achieve the energy efficiency. But there exists a tradeoff for the optimal design. For example, the protocol which aims to reduce idle monitoring and collisions always requires extra synchronizations and overheads, whereas, an increase in energy waste occurs due to collisions while reducing these overheads and synchronizations. 


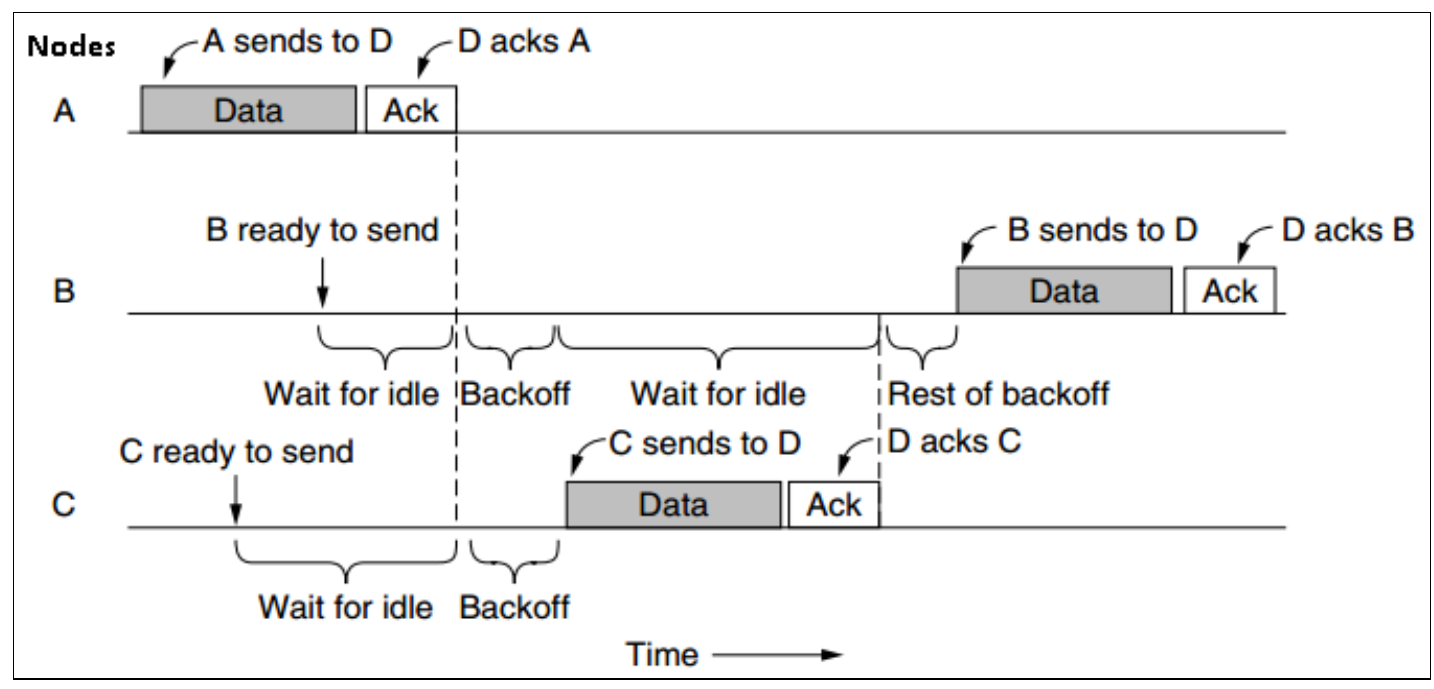

Fig 2: Sending a frame with MAC

That is why the proposal presented here focuses on the interfacing between the layers rather than implementing a protocol. This is a very simple method to implement which reduces the complexity and cost of the system along with the energy.

Several protocols are there to functionalize this sub-layer and among all of those, IEEE 802.11 MAC protocol tries to avoid collisions with a protocol called CSMA/CA (CSMA with Collision Avoidance) which can be found in [15]. Figure-2 illustrates the scenario clearly where it is shown that node A is the first to send a frame. While A is sending, nodes B and C become ready to send. They see that the channel is busy and wait for it to become idle. Shortly after A receives an acknowledgement, the channel goes idle.

However, rather than sending a frame right away and colliding, B and $\mathrm{C}$ both perform a backoff. $\mathrm{C}$ picks up a short backoff, and thus sends first. B halts its countdown while it senses that $\mathrm{C}$ is using the channel and resumes after $\mathrm{C}$ has received an acknowledgement. $\mathrm{B}$ soon completes its backoff and sends its frame.

This scheduling of MAC layer would be much more efficient if the information about the nodes' address and routing channel are provided to that very layer. We would like to use AODV routing protocol in the network layer as it stores a route table which consists of destination address, next hop address, destination sequence number and life time of any route. Our aim is to provide MAC layer with this route table which makes scheduling more efficient. When a node wishes to send a packet to some destination then it checks its routing table to determine if it has a current route to the destination. If it finds a root then it forwards the packet to next hop node or initiates a route discovery process if it is otherwise. Route discovery process begins with the creation of a Route Request (RREQ) packet which is created by source node. For each destination, a node maintains a list of precursor nodes, to route through them. Precursor nodes help in route maintenance. Life-time is also updated every time a route is used and if a route is never used within its life time then it expires. Therefore, energy will not be wasted. Say for example, B node has never been able to send any data to D. If the routing table is not there in the MAC layer, then this layer will schedule the data to be sent to D again. But again D will not be able to receive the data which will cause unnecessary loss of transmission energy.

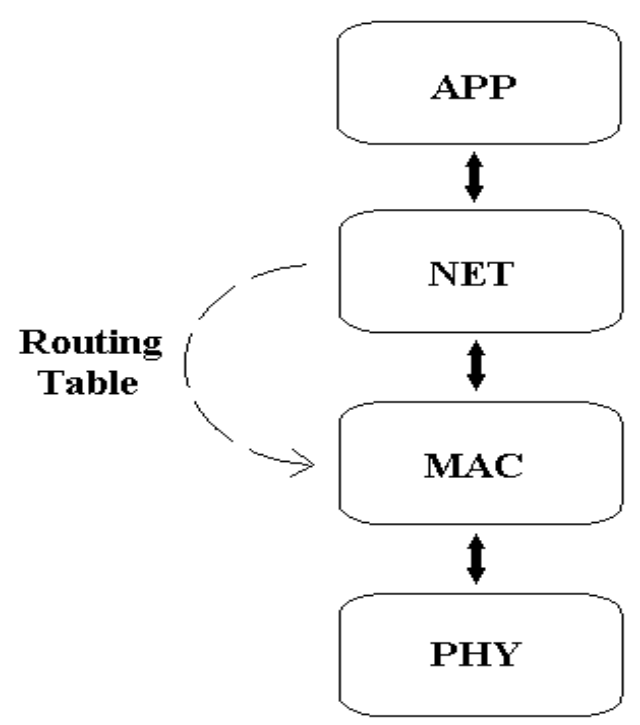

Fig 3: Overview of our cross-layer approach

Even if D receives the data from another node as WSN has that property, it will not send the data to its upper layer for further processing to communicate with $\mathrm{B}$ which will reduce the receiving energy as well. Rather D will turn into sleep mode if it never receives any data from any sensor node. Therefore our cross-layer approach will reduce energy consumption by controlling the flow of data throughout the network.

\subsection{Route Discovery}

When an intermediate node receives a RREQ, the node sets up a reverse route entry for the source node in its route table which consists of source IP address, source sequence number, number of hops to source node, IP address of node from which RREQ was received. Any node can send a RREP (Route Reply packet) to the source using the reverse route which also contains life time field. In order to respond to RREQ a node should have unexpired entry for the destination and sequence number of destination at least as great as in RREQ (for loop prevention) in its route table. If both conditions are met and the IP address of the destination matches with that in RREQ the node responds to RREQ by sending a RREP back using unicasting. If conditions are not satisfied, then node increments the hop count in RREQ and 
broadcasts to its neighbors. Ultimately the RREQ will make to the destination. Figure 4 to Figure 7 will explain the procedure in detail.

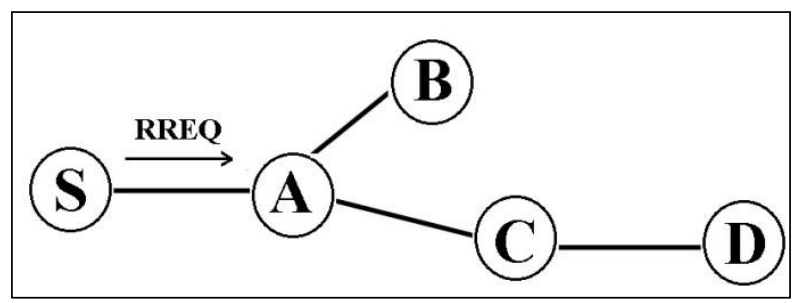

Fig 4: Node $S$ sends RREQ to Node A

From Figure 4 it can be seen that $S$ node needs a route to reach destination node $\mathrm{D}$. Therefore $\mathrm{S}$ creates a RREQ which enters D's IP address and sequence number, S's IP address and sequence number, hop count $(=0)$. Node $S$ broadcasts RREQ to neighbors and node A receives it which makes a reverse route entry for $S$ where, destination $=S$, next hop $=S$, hop count $=1$. A has no routes to D, so it rebroadcasts RREQ to its neighbors which is shown in Figure 5.

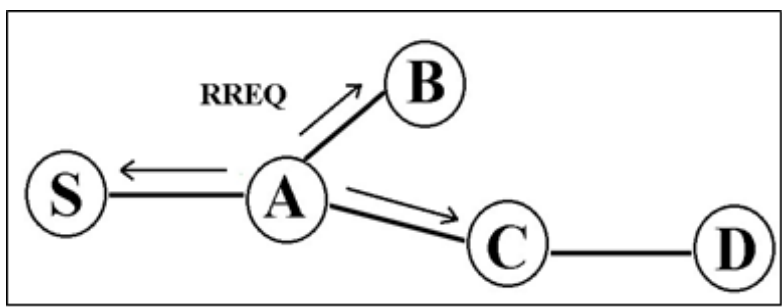

Fig 5: Node A sends RREQ to neighbors

Node $\mathrm{C}$ receives RREQ and makes a reverse route entry for $\mathrm{S}$ where, destination $=\mathrm{S}$, next hop $=\mathrm{A}$, hop count $=2$. As it has a route to $\mathrm{D}$ and the sequence for route to $\mathrm{D}$ is greater than D's sequence in RREQ, so C creates RREP. An intermediate node which knows a route with a smaller sequence number cannot send RREP. A new RREQ by node $\mathrm{S}$ for a destination is assigned a higher destination sequence number. C's RREP enters D's IP address and sequence number, S's IP address, hop count to $\mathrm{D}(=1)$.

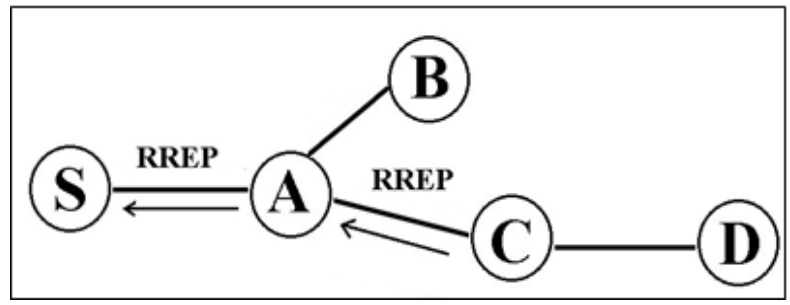

Fig 6: Node A sends RREP to Node $S$

Node A receives RREP and makes a forward route entry to D where, destination $=\mathrm{D}$, next hop $=\mathrm{C}$ and hop count $=2$. Then node A unicasts RREP to node $\mathrm{S}$. Any node may receive multiple RREP for a given destination from more than one neighbor. The node only forwards the first RREP it receives or may forward another RREP if that has greater destination sequence number or a smaller hop count. Rest of those are discarded which reduces the number of RREP propagating towards the source. Source can begin data transmission upon receiving the first RREP.

Now, after receiving RREP node $S$ makes a forward route entry to $\mathrm{D}$ where, destination $=\mathrm{D}$, next hop $=\mathrm{A}$, hop count $=$ 3 . Then it sends data packet to D where the route is S-A-C-D.
Broadcast transmission also follows the same procedure to send data packet to source to sink.

\subsection{Route Failure}

If the link between node $\mathrm{C}$ and node $\mathrm{D}$ breaks, then $\mathrm{C}$ creates Route Error (RERR) message rather creating a RREP and also invalidates the route to $\mathrm{D}$ in the routing table. RERR contains list of all destinations which are unreachable.

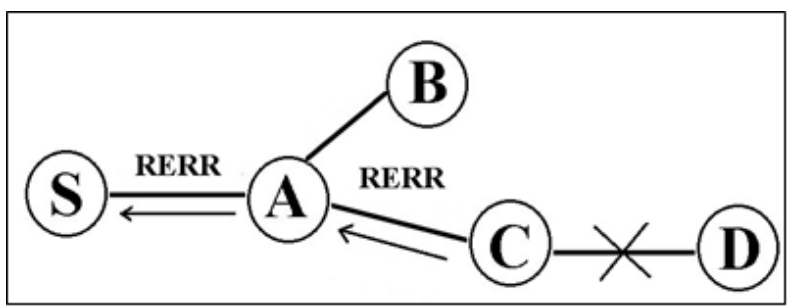

Fig 7: Link failure between node $\mathrm{C}$ and $\mathrm{D}$

When node A receives RERR, it checks whether $C$ is its next hop on route to $\mathrm{D}$ or not. Then it deletes route to $\mathrm{D}$ making distance to infinity and forwards RERR to node S. After receiving RERR node $S$ checks whether $A$ is its next hop on route to $\mathrm{D}$ and then deletes route to $\mathrm{D}$. It tries to rediscover another route if still needed. Unused routes automatically expire even if the topology does not change. Our cross-layer approach uses this route table constructed in the route discovery and route failure processes to be accessed by MAC layer to reduce the energy consumption throughout the network. As the number of sensor nodes has great impact in this method, therefore it would be rather logical to analyze the performance of our approach to the other protocols varying the number of nodes.

\section{PERFORMANCE EVALUATION}

\subsection{Simulation Parameters}

To analyze the performance of our cross-layer approach, we have used network simulator-2 (ns-2) software. This software can generate a real life networking scenario with ease. This is a discrete event simulator targeted at networking research which provides substantial support for simulation of Transmission Control Protocol (TCP), routing, and multicast protocols over wired and wireless (local and satellite) networks.

A clustered wireless network topology has been created with an event area of $800 \times 800 \mathrm{~m}^{2}$ where the source and sink nodes are also defined to several coordinates. The agents used in the transport layer are UDP (User Datagram Protocol) and Null agents as they do not use any acknowledgement while data transmission and thereby consume less energy than TCP agents. TCP ensures reliable data transmission with the acknowledgements they use after receiving a data or packet along with more energy consumption. As our research is mainly concerned about the energy issues, therefore, TCP agents are not used here. All the nodes then communicate with the sink node to establish the network. Then we have investigated several performance metrics varying the number of senor nodes. After that process we have applied our crosslayer approach to every sensor nodes and the same performance metrics are again being analyzed to make some comparative study with the other approaches. Number of nodes is varied in between 2 to 20 for every time the simulation is done.

Several simulation parameters are presented in Table 1. These values are kept unchanged for every simulation. 
Table 1. Simulation Parameters

\begin{tabular}{|c|c|}
\hline Parameters & Value \\
\hline Initial Energy & $10 \mathrm{~J}$ \\
\hline $\mathrm{PL}$ & $55 \mathrm{~dB}$ \\
\hline $\mathrm{E}_{\mathrm{rx}}$ & $13.5 \mathrm{~mW}$ \\
\hline $\mathrm{E}_{\mathrm{tx}}$ & $24.75 \mathrm{~mW}$ \\
\hline $\mathrm{E}_{\text {sleep }}$ & $1.5 \mathrm{~mW}$ \\
\hline $\mathrm{T}_{\text {transition }}$ & $16 \mathrm{~ms}$ \\
\hline Frame Length & $5 \mathrm{~s}$ \\
\hline Buffer Length & 30 \\
\hline Energy Threshold & $100 \mu \mathrm{j}$ \\
\hline CBR Packet Size & $512 \mathrm{~B}$ \\
\hline
\end{tabular}

The following performance metrics have been investigated in the evaluations:

- Energy Efficiency: This is one of the most important metrics in WSNs. In our analysis, this metric is definitely the most important one as we are focusing on reduction of energy consumption of the sensor nodes. We consider the total energy consumption of all the sensor nodes here. So, lower value of that indicates more energy efficiency.

- Goodput: It is defined as the ratio between the total number of unique packets received at the sink and the total number of packets sent by all the source nodes. This metric will ensure the communication reliability of the network. Higher value of it indicates more reliable communication.

- Throughput: It is defined as the bits per second received at the sink. We have considered all the packets here, not only the unique packets.

\subsection{Results}

Simulation results have been presented with the graphs shown in Figure 8, Figure 9, Figure 10, Figure 11, Figure 12 and Figure 13.

At first, total energy consumption (in joules) throughout the network is observed varying the number of nodes. Figure 8 shows the comparison between our cross-layer approach with the IEEE 802.11 MAC protocol and Figure 9 with the SMAC of different duty cycles.

From both of the figures it is clearly visible that, our crosslayer approach consumes less energy than traditional 802.11 MAC and S-MAC. S-MAC with $50 \%$ duty cycle means the node is active for half of the period and for the other half it remains in sleep mode. Therefore, it will definitely consume less energy than the S-MAC with $100 \%$ duty cycle which is clearly visible in Figure 9. But our cross-layer approach outperforms both of those considering the fact of energy consumption throughout the network. The comparison gets more visible for increasing the number of sensor nodes. Therefore, our cross-layer approach works significantly for the larger sized sensor networking scenario.

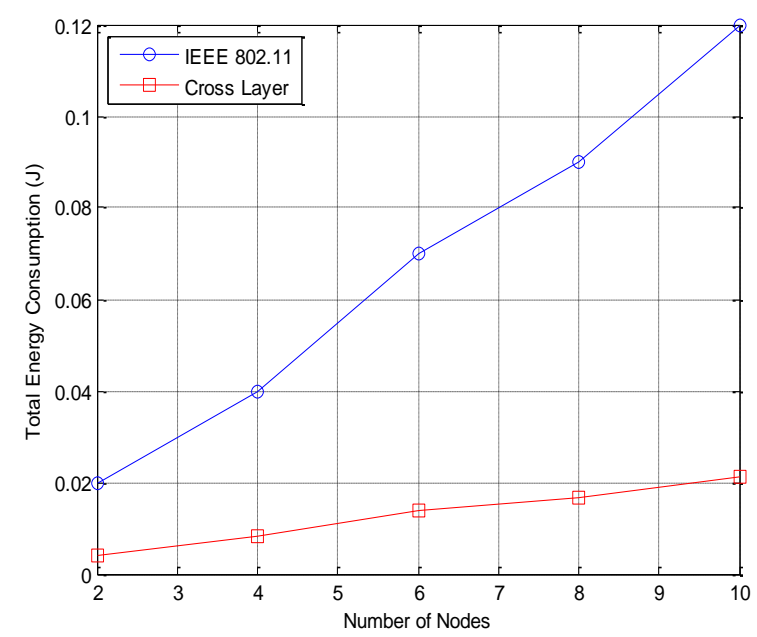

Fig 8: Energy Comparison between IEEE 802.11 MAC and Cross-layer Approach

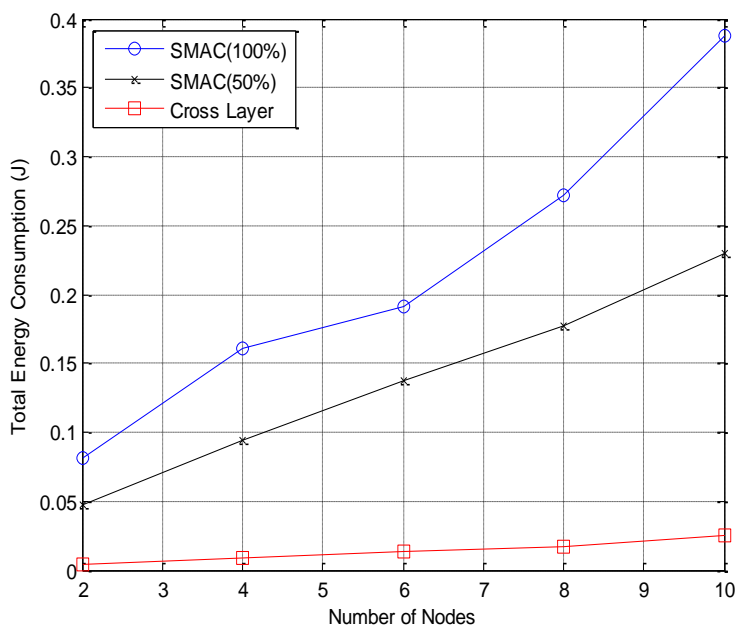

Fig 9: Energy Comparison between S-MAC and Crosslayer Approach

Then the goodput analysis is presented in Figure 10 and Figure 11. Goodput is the ratio of data received to data transmitted or sent by a node. It is clearly observed from Figure 10 that, our approach not only consumes less energy but it also maintains a good number of data receptions to transmissions ratio. For several nodes goodput reduces to IEEE 802.11 as because our cross-layer approach controls the data flow to save energy. Although then it performs well considering the amount of energy it consumes and maintaining a good throughput as well which is visible in Figure 11. 


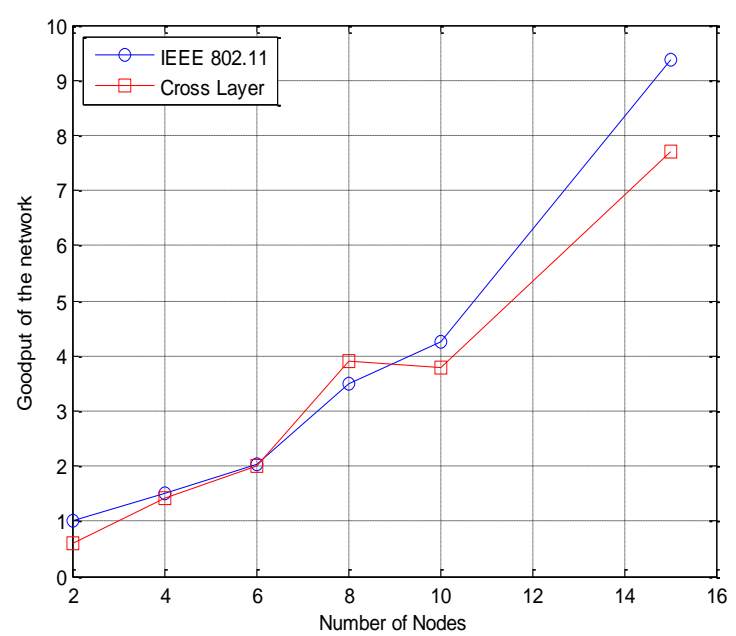

Fig 10: Goodput Comparison between IEEE 802.11 MAC and Cross-layer Approach

So from Figure 10, it can be concluded that the proposed approach can have less goodputs in several points of the graph than IEEE 802.11 MAC, but it receives more data throughout the simulation time which is shown in Figure 11. Therefore, this little bit of trade-off could easily be dealt with considering the overall performance.

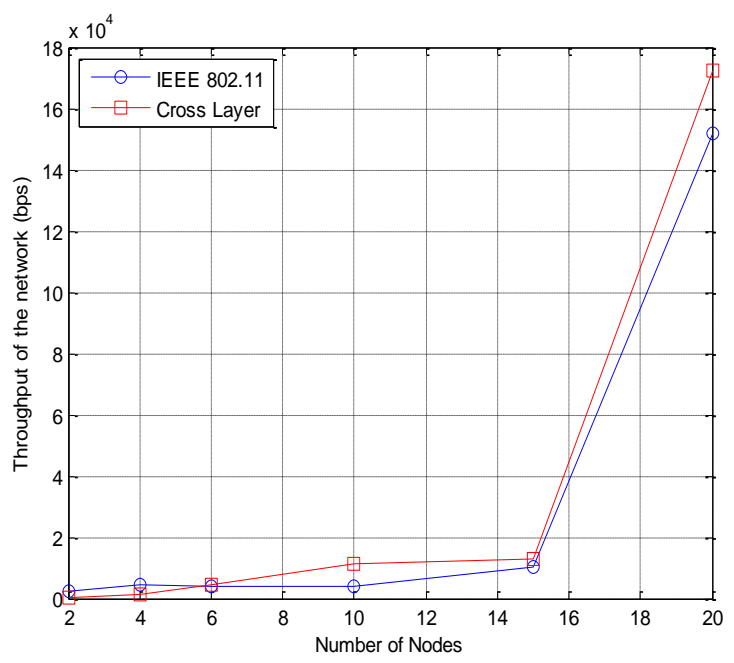

Fig 11: Throughput Comparison between IEEE 802.11 MAC and Cross-layer Approach

The goodput comparison between our cross-layer approach and S-MAC with different duty cycles has also been taken into consideration which can be found in Figure 12. S-MAC with duty cycles of $100 \%$ and $50 \%$ are considered only for our analysis. $50 \%$ of duty cycle denotes that the node is active for $50 \%$ of the time and remains off for the other $50 \%$ of the time.

To investigate the individual effect of our cross-layer approach for IEEE 802.11 MAC and S-MAC in terms of energy efficiency, the percentage of energy efficiency is also calculated which is illustrated in Figure 13. This is done by comparing the energy of our approach to the energy of IEEE 802.11 MAC or S-MAC and then taking ratio to get the percentage.

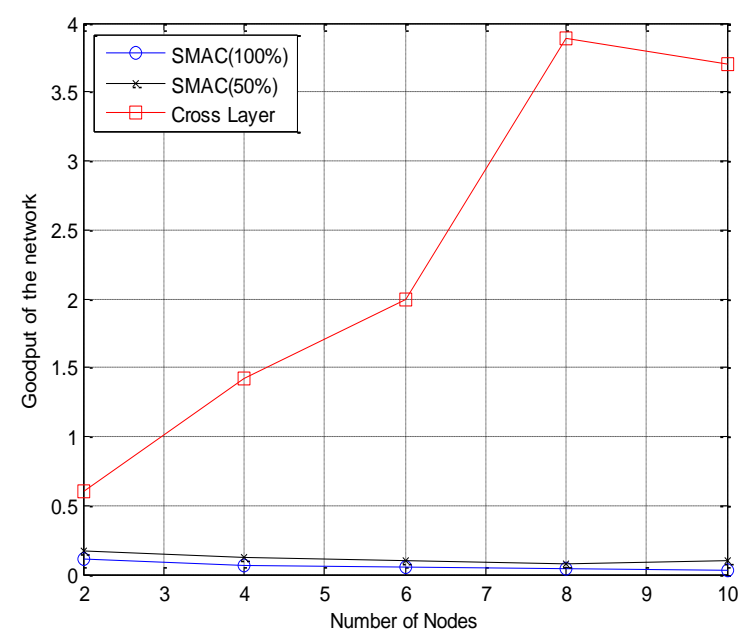

Fig 12: Goodput Comparison between S-MAC and Crosslayer Approach

Figure 12 suggests that S-MAC maintains a poor number of data receptions and transmission ratio for its duty cycle operation and it is totally outperformed by our cross-layer approach

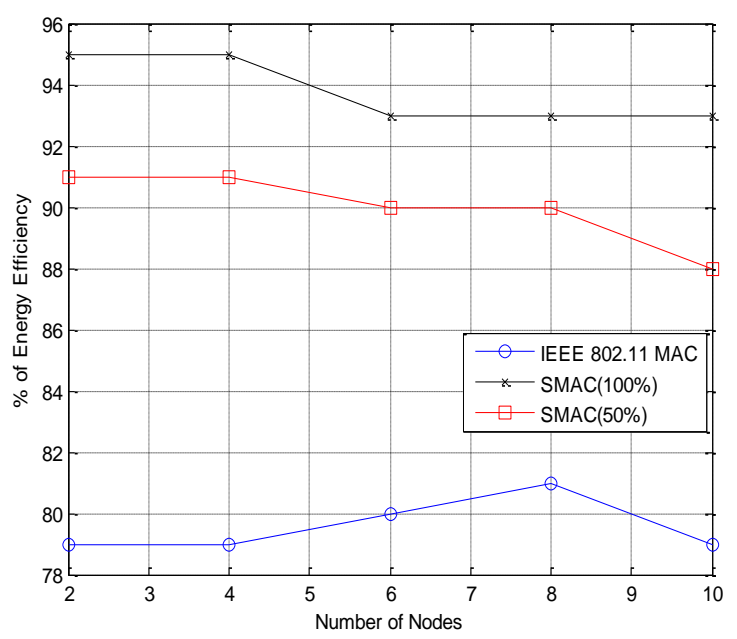

Fig 13: Percentage of Energy Efficiency Calculation

Therefore, from Figure 13, it can be seen that our cross-layer approach is more energy efficient for S-MAC of $100 \%$ duty cycle comparing with S-MAC of 50\% duty cycle and IEEE 802.11 MAC while the routing protocol used is AODV.

In addition to the performance regarding simulation issues, the implementation issues are also very important. Our crosslayer approach does not necessarily require any external hardware or complex circuitries, rather a simple technique inside the software makes the interfacing between the two layers stronger and thus the efficiency is achieved.

\section{CONCLUSION}

This paper tries to represent that traditional layered protocol can still be efficient if cross-layer approach is performed in it. It has made IEEE 802.11 MAC more energy efficient just crossing it with the network layer and result shows that it outperforms S-MAC as well which is more energy efficient than IEEE 802.11 MAC. It also reduces the complexity and 
expenditure of implementing a new protocol inside sensor network.

The ultimate goal of our cross-layer approach is to utilize the resource of one layer to another layer. AODV routing protocol uses route table to every nodes, which leads to more energy consumption along with the implementation complexity when works with the MAC layer of IEEE 802.11 module. But it can be seen that combining these two layers with a little effort using our cross-layer approach can have a significant impact for several performance metrics of the network. In traditional layered structures, like in TinyOS [16], the upper layers have to wait for the lower layers to process the data as because a single buffer is used to process data or packet for all the layers. This may lead to significant energy consumption if the packet needs not to be processed further. This scheduling can be done more efficiently if the access of the routing table is given to the MAC layer from the network layer.

Our research deals with the IEEE 802.11 platform now, but the future work of it includes the IEEE 802.15.4 platform which is one of the latest modules.

\section{REFERENCES}

[1] M. C. Vuran, O. B. Akan, and I. F. Akyildiz, "SpatioTemporal" Correlation: Theory and Applications for Wireless Sensor Networks", Computer Networks Journal (Elsevier), Vol. 45, No. 3, pp. 245-261, June 2004.

[2] O. B. Akan, Ian F. Akyildiz, "Event-to-Sink Reliable Transport in Wireless Sensor Networks", IEE/ACM Transactions on Networking, vol. 13, No. 5, pp. 10031016, Oct. 2005.

[3] C. Intanagonwiwat, R. Govindan, D. Estrin, J. Heidemann, and F. Silva, "Directed diffusion for wireless sensor networking", IEEE/ACM Trans. on Networking, vol. 11, no. 1, pp. 2 - 16, February 2003.

[4] K. Seada, M. Zuniga, A. Helmy, and B. Krishnamachari, "Energy-efficient forwarding strategies for geographic routing in lossy wireless sensor networks", in Proc. ACM Sensys 04, November 2004

[5] L. van Hoesel, T. Nieberg, J. Wu, and P. J. M. Havinga, "Prolonging the lifetime of wireless sensor networks by cross-layer interaction", IEEE Wireless Communications, vol. 11, no. 6, pp. 78 - 86, December 2004

[6] C. Bennila Thangammal, P. Rangarajan and J. Raja Paul Perinbam, "Maximization of Wireless Sensor Network's
Lifetime using Losningen Cross-layer Approach", Asian Journal of Scientific Research, 5, pp. 133-142, May 2012.

[7] J. Hapoola, Z. Shelby, C. Pomalaza-Raez, and P. Mahonen, "Cross-layer energy analysis of multi-hop wireless sensor network", in EWSN' 05, 2005, pp. 3344

[8] Luca Catarinucci, Riccardo Colella, Giuseppe Del Fiore, Luca Mainetti, Vincenzo Mighali, Luigi Patrono, and Maria Laura Stefanizzi, "A Cross-Layer Approach to Minimize the Energy Consumption in Wireless Sensor Networks", International Journal of Distributed Sensor Networks, vol. 2014, Jan. 2014

[9] Yuebin Bai, Shujuan Liu, Mo Sha, Yang Lu, and Cong $\mathrm{Xu}$, "An Energy Optimization Protocol Based on CrossLayer for Wireless Sensor Network", Journal of Communications, vol. 3, No. 6, pp. 27-34, Nov. 2008.

[10] Chia-Hung Tsai, Tsu-Wen Hsu, Meng-Shiuan Pan, and Yu-Chee Tseng, "Cross-Layer, Energy-efficient Design for Supporting Continuous Queries in Wireless Sensor Networks: A Quorum-Based Approach", Wireless Personal Communications, vol. 51, Issue 3, pp. 411-426, July 2009.

[11] Mehmet C. Vuran, Ian F. Akyildiz, "XLP: A CrossLayer Protocol for Efficient Communication in Wireless Sensor Networks", IEEE Transactions on Mobile Computing, vol. 9, No. 11, pp. 1578-1591, Nov. 2010.

[12] Ian F. Akyildiz, Mehmet C. Vuran, and Ozgur B. Akan, "A Cross-Layer Protocol for Wireless Sensor Networks", Proc. Conf. Information Sciences and Systems (CISS '06), pp. 1102-1107, Mar. 2006.

[13] Wei Ye, John Heidemann, and Deborah Estrin, "Medium Access Control With Coordinated Adaptive Sleeping for Wireless Sensor Networks", IEEE/ACM Transactions on networking, vol. 12, No. 3, pp. 493-506, June 2004.

[14] Muhammad Mahtab Alam, Olivier Berder, Daniel Menard, Thomas Anger, and Olivier Sentieys, "A Hybrid Model for Accurate Energy Analysis of WSN Nodes", EURASIP Journal on Embedded Systems, vol. 2011, pp. 1-17, January 2011.

[15] Andrew S. Tanenbaum, David J. Wetherall, "Computer Networks" ( $5^{\text {th }}$ edition), page. 303-304.

[16] TinyOS, http://www.tinyOS.net, 2010. 